\title{
DEL PREGUNTARIO DE LOS NIÑOS: DE LA ACTIVIDAD DE LOS MICROBIOS MÁS ALLÁ DE LA GOTA DE LECHE
}

\section{WOULD ASK OF THE CHILDREN: THE ACTIVITY OF MICROBES BEYOND THE DROP OF MILK}

\author{
POR: Silvio Fernando Daza Rosales ${ }^{1}$ \\ Mario Roberto Quintanilla Gatica² \\ José Rafael Arrieta Vergara ${ }^{3}$ \\ Oswaldo Ríos Carrascal ${ }^{4}$
}

Realmente existe en la naturaleza un cuarto reino: el de la imaginación. Gracias a esa maravillosa realidad podemos conocer la verdadera esencia de los gatos y de sus hermanos los búhos, de los conejos y su predilección por el cultivo de la zanahorias, de las ovejas y sus nubes de lana, de los caballos voladores, en fin, la esencia de todos los seres que habitan el cuarto reino de la naturaleza. Jairo Aníbal Niño (1989)

"La ciencia no nos habla de la naturaleza: nos ofrece respuestas a nuestras preguntas sobre la naturaleza. Lo que observamos no es la naturaleza en sí misma, sino la naturaleza a través de nuestros modos de preguntar" W. Heisenberg (1972)

\section{Resumen}

Esta investigación de tipo descriptivo, específicamente encajado como estudio de caso, desarrollada con 80 niños de la institución educativa Diego Hernández de Gallegos de Barrancabermeja, Colombia, tuvo como objetivo construir conocimiento científico escolar desde el conocimiento cotidiano y científico a través de un ejercicio con un producto como la leche; para lograr esto se desarrolló con los estudiantes, una experiencia con la diferencia de perecibilidad entre la leche en caja y la leche en bolsa. Donde los niños a partir del planteamiento de una situación

${ }^{1}$ Escuela de Agronomía. Instituto Universitario de la Paz-Colombia y Director del Grupo de Investigación para la Renovación de la Enseñanza de las Ciencias (GRECI) biosidaza52@hotmail.com

${ }^{2}$ Profesor de la Facultad de Educación Pontificia Universidad Católica de Chile y director del Laboratorio de Investigación en Didáctica de las Ciencias (GRECI)

mariorqg@gmail.com

${ }^{3}$ Escuela de Agronomía. Instituto Universitario de la Paz-Colombia y director del grupo de investigación de cultivos tropicales (INYUBA)

llanadero@hotmil.com

${ }^{4}$ Escuela de Agronomía. Instituto Universitario de la Paz-Colombia y director de la Red de semilleros locales de investigación en Barrancabermeja (REDCOLCI)

oswy62@hotmail.com 
problema, formularon preguntas, propusieron hipótesis y diseños experimentales y concluyeron desde la información bibliográfica, para finalmente resolver una situación de contexto. Se puede concluir que es una experiencia donde los estudiantes a partir de su participación activa y consensuada en el aula de clases, construyen conocimiento escolar. Se desarrolla una enseñanza desde la cotidianidad que los impregna de un significado de experiencia de vida que los motiva (más temprano que tarde) a la ciencia y la investigación.

\section{Palabras clave:}

Pregunta, comunidad de indagación, competencia de pensamiento científico, trabajo colaborativo, investigación orientada.

\section{Abstract}

This research from descriptive, specifically embedded type as a case study developed with 80 children from the educational institution Diego Hernández Gallegos of Barrancabermeja, Colombia, aimed to build school scientific knowledge from everyday and scientific through an exercise with a product knowledge as milk; to achieve this was developed with students, experience with the difference of perecibility between boxed milk and milk bag. Where children from the approach to a problem situation, questions, proposed hypotheses and experimental designs and concluded from the bibliographic information, to finally resolve a situation of context. It can be concluded that it is an experience where students from their active participation and consensus in the classroom, construct school knowledge. Develops a teaching from everyday life that pervades them a meaning of life experience (sooner rather than later) motivating them to science and research.

\section{Key words:}

Question, a community of inquiry, scientific thinking skills, teamwork, research-oriented.

\section{Introducción}

La calidad de nuestras vidas determina la de nuestras preguntas, ya que estas son la maquinaria, la fuerza que impulsa el pensamiento (Linda, E. et al, 2002). Sin ellas, no tenemos sobre qué pensar. Sin las preguntas esenciales, muchas veces no logramos enfocar el pensar en lo significativo y sustancial. Las preguntas son la base de la actividad mental de razonar y comprender el problema y el estímulo fundamental que utiliza el 'observador' para mantener en actividad al 'resolvedor' (Lockhead, 1987).

Las preguntas juegan un papel fundamental, ya que definen las tareas, expresan problemas y delimitan asuntos, impulsan hacia adelante el pensar. Las contestaciones, por otra parte, a menudo indican una pausa en el pensar. Una contestación genera otras preguntas que hacen que el pensamiento continúe la indagación. Una mente sin preguntas no está viva intelectualmente. El no hacerlas equivale a no comprender y las superficiales equivalen a poca comprensión y las poco claras equivalen a comprensión no clara. Si la mente no las genera, no hay aprendizaje sustancial (Elder y Paul, 2002).

El buen preguntario en los niños, está dado generalmente como respuesta a ligeros estímulos concretos como una historieta, una fábula, un cuento, un fenómeno de las ciencias naturales, un poema, un dibujo o un comic, un juego, etc. No es difícil animar a los niños para que formulen preguntas, ya que ellos están interesados de manera natural en algunas 
cosas, y cuando lo están, aumentan de forma notable el número y la diversidad de las preguntas que hacen (Ogborn, J. et al 1998), a menos que el trato irreflexivo de los adultos les haya hecho desistir de dar rienda suelta a su imaginario de inclinaciones naturales. El docente juega un papel trascendental en hacer resurgir con bastante facilidad las preguntas de los niños, especialmente si todas ellas reciben adecuada respuesta y no solo las que el docente considera importante.

Harlen (1999), plantea "que en el aprendizaje de los niños, es muy importante que se susciten gran cantidad de preguntas, incluso las no muy correctamente expresadas y las que resultan vagas en exceso, porque las preguntas constituyen el medio por el cual el niño puede enlazar unas experiencias con otras, facilitándole la construcción de su propia imagen del mundo".

Para Duckwoorth (1999), la pregunta adecuada en el momento correcto puede llevar al niño a alcanzar picos en su pensamiento, que den como resultado adelantos muy significativos y una verdadera estimulación intelectual, si el ámbito en el que se mueve es el adecuado; son los mismos niños los que pueden llegar a formularse la pregunta orientadora. Una vez generada la pregunta, se movilizan las ideas para poner a prueba al máximo la capacidad para encontrar la respuesta. Lo que podemos hacer es que los niños entren en contacto con ciertos fenómenos biológicos, químicos, físicos y tecnológicos, de modo tal que se pueda captar sus interés, dejarlos generar y responder sus propias preguntas, y permitirles darse cuenta de que sus ideas son importantes, para que tengan el interés, la habilidad y la confianza en sí mismos para seguir avanzando.

Las preguntas juegan papeles estratégicos en la enseñanza de las ciencias como comprensión científica del mundo. Para esto se hace necesario pedir a los niños que clarifiquen sus propias ideas y creencias acerca de un fenómeno o problema particular; la mejor solución es ofrecerles la oportunidad de participar en conversaciones en las que se tomen en serio sus experiencias y percepciones del mundo; alentarlos a ver que son posibles puntos de vista alternativos instándolos a adoptar diferentes perspectivas, por ejemplo presentar el punto de vista científico probado como uno más entre otros, y compararlo con otros posibles respecto de la fuerza explicativa, relevancia, consistencia, plausibilidad; requerir que den razones de sus puntos de vista y que el docente proporcione razones para aceptar puntos de vista alternativos; invitarlos a autocorregirse por ejemplo, planteando el problema en cuestión en un contexto histórico apropiado.

Se hace necesario entonces, construir una comunidad de indagación donde los niños observen, planteen preguntas y experimenten con objetos y fenómenos reales y cercanos. Razonen, discutan, compartan ideas y construyan conocimiento. Las actividades deben organizarse en secuencias que guíen la indagación pero también que dejen espacio para que los niños se desenvuelvan con autonomía. Aquí aprendizaje y enseñanza de las ciencias han de desarrollarse como un proceso de (re)construcción de conocimientos, en un contexto que se inspire en la metodología de la investigación como forma de favorecer, tanto una actividad significativa en torno a problemas susceptibles de interesar a los niños, como en su progresiva autonomía de juicio y capacidad de participación en tareas colectivas.

El aprendizaje cooperativo se pone en práctica en pequeños grupos de trabajo, para animar a los niños a que hagan preguntas de cualquier tipo.

Para desarrollar estas capacidades es necesario que el docente propicie los comentarios entre los propios niños, que dé tiempo para que ellos discutan sus diferencias y compartan 
sus conocimientos e ideas sobre los fenómenos naturales. Para ello es conveniente tener en clase colecciones de objetos nuevos, llevar a los niños de paseo o de visita, proporcionarles gran variedad de materiales para que los manipulen. Aquí los niños tienen roles o tareas bien definidas, y la responsabilidad global por el progreso es compartida entre todos sus miembros en una gama de actividades: desde hablar, preguntar, escuchar, escribir, leer, dibujar, el preguntar y el escuchar entrelazado con otras actividades. Actividades que permitan a los niños escucharse, construir ideas unos con otros aportando y analizando razones en sustentos de los argumentos propuestos; ayudándose a formular preguntas y ampliar los puntos de vista desafiando la del otro con un contraejemplo; dando tiempo y espacio para que la voces se expresen por sí misma.

Para lograr cambios profundos hay que buscar el interés en los niños por un modelo de investigación dirigida, que permita transformar en su mente, no solo en lo conceptual sino también en lo procedimental, actitudinal y en lo ontológico. Para esto es preciso situarlos en un contexto de actividad similar o análoga al que vive un científico guardando las proporciones de finalidad y contextos. Así como la metáfora de Gil D. et al (2002), en la que considera a los niños como investigadores noveles proporciona una mejor apreciación de la situación de aprendizaje, en donde se pretende desarrollar el pensamiento crítico, ético, estético... en definitiva, la utopía en la que todo niño tiene derecho a iniciarse. El reto es, precisamente, que enseñar-aprender ciencias constituya una actividad escolar que tenga como resultado la construcción de conocimiento dinámico, es decir, que pueda transformar también el mundo de los alumnos haciéndoles capaces de intervenir en el mundo y de tomar decisiones.

La propuesta de organizar el aprendizaje de los niños como una construcción de conocimientos, responde a la de una investigación orientada con resultados parciales, embrionarios, obtenidos por la "comunidad científica". No se trata de "engañar" a los niños, de hacerles creer que los conocimientos se construyen con la aparente facilidad con que ellos los adquieren (Gil, D., 2002), sino de ponerlos en una situación por la que los científicos habitualmente pasan durante su formación, y en la que podrán familiarizarse mínimamente con lo que es el trabajo científico y sus resultados, replicando para ello investigaciones ya realizadas por otros, abordando, en definitiva, problemas conocidos por quienes dirigen su trabajo.

La idea de que la enseñanza y el aprendizaje de las ciencias se desarrollen como un proceso de (re)construcción de conocimientos en un contexto que se inspire en la investigación, significa que en todos los niveles la educación científica debe basarse en la metodología de la investigación como forma de favorecer una actividad significativa, en torno a problemas susceptibles de interesar a los niños, como en su progresiva autonomía de juicio y capacidad de participación en tareas colectivas, (Osborne y Wittrok, 1983; Duschl, 1995; Gil, D. et al., 1999). El contexto hipotético deductivo característico de una investigación suministra oportunidades idóneas para un aprendizaje profundo (Pozo, J. 1989), al obligar a plantear problemas y discutir su relevancia, tomar decisiones que permitan avanzar, formular ideas de manera tentativa, ponerlas a pruebas dentro de una estructura lógica general, obtener evidencias para apoyar las conclusiones, utilizando los criterios de coherencia y universalidad, y todo ello en un ambiente de trabajo colectivo y de implicación personal en la tarea. 
Para que esto pueda llevarse a cabo, Daza S. y Arrieta, R., (2006) indican que el desarrollo de un tema no puede responder a la lógica tradicional de la clase y a un orden predeterminado de preguntas como: ¿Qué objetivo deben lograr los estudiantes? ¿Qué contenidos impartir? ¿Cómo ha de ser el examen para constatar el aprendizaje logrado?, sino que deben formularse preguntas tales como: ¿Cómo problematizar el curso y cada uno de los temas incluidos, para favorecer el aprendizaje con sentido? ¿Cómo evaluar para impulsar y orientar dicho aprendizaje?

El desarrollo debe guiarse por preguntas como: ¿Qué problemas están en el origen de las teorías que deseamos que pasen a formar parte de nuestros niños? ¿Cuáles son/fueron los obstáculos más importantes que hubo que superar para avanzar en la solución de los problemas planteados? ¿Qué ideas y razonamientos pueden tener los niños que puedan suponer obstáculos para el aprendizaje y que, por tanto, deban ser tomados en consideración? ¿Qué plan concreto de investigación-secuenciación conviene proponer a los niños para avanzar en la solución de los problemas iníciales?

Por lo tanto se propone una estructura que permita a los niños enfrentarse a situaciones problemáticas de interés, teniendo en cuenta los procesos de producción y validación de los conocimientos científicos (Martínez T. et al, 2003) como se muestra a continuación.

- $\quad$ Plantear al inicio del curso, situaciones problemáticas que inspirándose en las que están en el origen de los conocimientos implicados sirvan de punto de partida para despertar el interés por preguntar de parte de los niños. Debe prestarse atención a la apropiación de los problemas por parte de ellos y a que tomen conciencia para el desarrollo de las tareas.

- Diseñar la secuenciación de los temas del curso con una lógica problematizada, como estrategia para avanzar en la solución de las preguntas iniciales. Cada tema se convierte en un problema más concreto, cuya solución permite avanzar en el problema inicial y al tiempo generar nuevos problemas.

- $\quad$ Organizar el índice de cada uno de los temas/problemas, de forma que responda a una estrategia para avanzar en su solución o a un "plan de investigación" diseñado por el docente (o, mejor, por el equipo de docentes). La estructura o secuencia de apartados debe estar ligada intencional y lógicamente a la problematización inicial. Pero la estructura no está guiada por los conceptos fundamentales, sino por un modo de plantear y avanzar en los problemas fundamentales. Los conceptos son introducidos como parte del proceso de tratamiento de los problemas planteados y de unificación de campos inicialmente inconexos. Si el conocimiento es científico, es fruto de un intento de responder las preguntas.

- Introducir los conceptos y modelos como hipótesis fundadas, que deben ser puestas a prueba en situaciones de laboratorio y/o en el aborde de soluciones problemáticas abiertas concretas, que requieren una modelización, basadas en los mismos (contexto de resolución de problemas, incluyendo la toma de decisiones en situaciones de interés social), como a través del establecimiento de su coherencia con la globalidad de los conocimientos ya establecidos por investigaciones precedentes (Gil D. et al., 1999). 
- $\quad$ Realización de recapitulaciones periódicas problematizadas sobre los avances, los obstáculos superados y lo falta por hacer, prestando especial atención a la orientación de los niños en el desarrollo de la investigación.

- Explicitar las propias ideas y confrontarlas con las de otros autores, en un proceso hipotético deductivo.

Se pretende así, crear un ambiente que favorezca simultáneamente la implicación afectiva y la racionalidad científica de todos los implicados, (los niños, docentes y padres de familia) en la resolución de los problemas.

\section{Materiales y métodos}

Ubicación: esta actividad se desarrolló en la institución educativa Diego Hernández de Gallegos, de Barrancabermeja, Colombia,

Población: se trabajó con una población total de 80 niños de la jornada de la mañana, procedentes en su mayoría de barrios de estratos 1 y 2, cercanos a la institución. El grupo se dividió en dos subgrupos de 40 niños con edades entre 9 y 11. Buscó analizar cuál era la actitud de los niños cuando se enfrentaban a una situación cotidiana.

Tipo de investigación: fue un estudio de caso, del tipo descriptivo, donde no hubo manipulación de variables; la información se tomó de fuentes secundarias y primarias, se clasificó y ordenó en tablas y graficas para su posterior análisis.

\section{Desarrollo de las actividades}

Planteamiento de la situación problema: se inició planteando una situación problema de la vida cotidiana que despertó el interés en los niños por resolverla, explicitando ideas de lo que conocían; de este modo ocuparon el lugar de poseedores de conocimientos, desplegando su confianza para plantear y discutir posibles respuestas, adjudicándole autoridad e importancia al conocimiento popular o extraescolar que ellos poseían respecto al tema tratado. En el Cuadro 1, se muestra la actividad central, la revisión de las etiquetas de las bolsas y cajas de leche y se buscó que los niños propusieran hipótesis y trataran de explicar la diferencia de perecibilidad de la leche en bolsa y en la caja. 


\section{Cuadro 1. La actividad de la unidad didáctica: los microbios más allá de la gota de leche.}

\begin{tabular}{|c|c|}
\hline $\begin{array}{l}\text { Actividad central: } \\
\text { Revisión de las } \\
\text { etiquetas de las bolsas }\end{array}$ & $\begin{array}{l}\text { Agrupación. Equipos de } \\
\text { trabajo cooperativo }\end{array}$ \\
\hline $\begin{array}{l}\text { inalidades: } \\
\text { Propone hipótesis y } \\
\text { xplica la diferencia en } \\
\text { I tiempo de duración } \\
\text { e la leche empacada } \\
\text { n caja y bolsa. } \\
\text { dentifica los términos } \\
\text { esconocidos y la } \\
\text { omposición química de } \\
\text { leche en los } \\
\text { mpaques de caja de } \\
\text { artón y bolsa plástica. }\end{array}$ & $\begin{array}{l}\text { Contenidos: } \\
\text { Composición de la leche. } \\
\text { Características del empaque de la leche (bolsa y caja). } \\
\text { Pregunta orientadora: } \\
\text { ¿Por qué la leche empacada en caja dura más que la } \\
\text { empacada en bolsa? }\end{array}$ \\
\hline \multicolumn{2}{|c|}{$\begin{array}{l}\text { Por grupos, los niños debían llevar al aula de clase una caja y una bolsa de leche vacía, } \\
\text { oara analizar las etiquetas y dar respuesta a las siguientes preguntas: ¿Por qué la leche } \\
\text { en caja dura más que la de la bolsa? A partir de la pregunta orientadora los niños } \\
\text { sugirieron las siguientes preguntas: ¿De qué está hecha la caja y la bolsa para que dure } \\
\text { más la leche sin dañarse? ¿Qué contiene de diferente la leche de la caja con la de bolsa } \\
\text { que hace que ella dure más? ¿Por qué la leche de caja tiene mayor tiempo de } \\
\text { vencimiento que la de bolsa? De ahí debían surgir hipótesis, susceptibles de ser } \\
\text { comprobadas. Para esto los estudiantes elaboraron diseños experimentales, para así } \\
\text { descartar las hipótesis o respuesta tentativa y dejar la más coherente. Además, } \\
\text { dentificaron diversos términos relacionados con la conservación de la leche, registrados } \\
\text { en cada una de las etiquetas, para ser comentados en la actividad siguiente. } \\
\text { Regulación: Implementación de la estrategia de resolución de problemas como } \\
\text { nvestigación dirigida, en situaciones de la vida cotidiana, relacionadas con la diferencia } \\
\text { en la duración de la leche empacada en cajas y en bolsa. Consulta de términos } \\
\text { desconocidos hallados en la etiqueta de la leche. }\end{array}$} \\
\hline & \\
\hline
\end{tabular}

Formulación de preguntas iniciales: se les preguntó ¿por qué la leche empacada en caja dura más que la empacada en bolsa?

Y las respuestas fueron:

"La leche de caja dura más que la de bolsa porque le echan un químico. Hay más químicos en la leche de caja".

$>\quad$ "La leche de caja está hecha de varias capas como son: cartón, plástico y papel aluminio y la de bolsa solo es un plástico que por dentro está pintado de color negro".

$>\quad$ "La leche de caja dura más porque pasa por fábricas, la limpian y descontaminan más".

$>\quad$ "La caja está hecha de un material más resistente y duradero". 
Planteamiento de hipótesis: con estas respuestas, se propusieron las siguientes hipótesis o conjeturas:

Primera hipótesis: la leche de caja dura más que la leche en bolsa porque le echan un químico.

Segunda hipótesis: la leche de caja dura más que la leche en bolsa porque está mejor sellada (tetra pack); tiene tres empaques para evitar que le entren microbios y la bolsa de leche, aunque esté sellada, tiene pequeños agujeritos que no se ven a simple vista y por ahí entran los microbios.

Tercera hipótesis: la leche de caja tiene más proceso y está mas purificada (pasteurizada, esterilizada, ultrapasteurizada e higienizada) que la leche en bolsa.

Planteamientos de los diseños: a continuación se les preguntó a los niños Cómo iban a demostrar cuál de las tres hipótesis era la más aceptable

Los niños respondieron que se deberían plantear diseños experimentales para cada una de las hipótesis formuladas, que se muestran a continuación:

Diseño experimental para la primera hipótesis: se consultó en Internet la composición química en ambos empaques de leche, para mirar que químicos diferentes tenían. Se observó la composición química de la leche en las cajas y en las bolsas y se comparó con la información tomada de Internet, para observar si existían químicos que se añadían a la leche en caja para que se conservara más que la leche en bolsa. Los niños compararon lo leído en Internet con la composición química mostrada en las etiquetas. Para guiar el trabajo, los docentes preguntaron: ¿De qué está hecha la leche? Esta debía ser resuelta con el ejercicio de revisión y comparación de las tablas de composición de la leche tomada de Internet y de las halladas en las etiquetas.

Cuadro 2. Composición de la leche.

\begin{tabular}{|l|l|l|l|}
\hline \multicolumn{1}{|c|}{ ELEMENTOS } & \multicolumn{1}{c|}{ CANTIDAD } & ELEMENTOS & \multirow{2}{*}{ CANTIDAD } \\
\hline Calorías & 59 a $65 \mathrm{Kcal}$ & Fósforo & $90.00 \mathrm{mg}$. \\
\hline Agua & 87 a $89 \%$ & Sodio & $30 \mathrm{mg}$. \\
\hline Carbohidratos & 4.8 a $5 \mathrm{gr}$. & Fósforo & $90 \mathrm{mg}$. \\
\hline Grasas & 3 a $3.1 \mathrm{gr}$. & Potasio & $142 \mathrm{mg}$. \\
\hline Proteínas & $3.50 \mathrm{gr}$. & Calcio & $125 \mathrm{mg}$. \\
\hline Fibra & $0.00 \mathrm{gr}$. & Magnesio & $8 \mathrm{mg}$. \\
\hline Colesterol & $14.00 \mathrm{mg}$. & Cobre & $0.03 \mathrm{mg}$. \\
\hline Hierro & $0.2 \mathrm{mg}$. & Calcio & $125.00 \mathrm{mg}$. \\
\hline Vitamina A & $0.2 \mathrm{mg}$. & Vitamina B2 & $0.2 \mathrm{mg}$. \\
Vitamina B1 & $0.1 \mathrm{mg}$. & Vitamina B3 & $0.2 \mathrm{mg}$. \\
\hline
\end{tabular}

Fuente: $\underline{\text { http://www.zonadiet.com/bebidas/leche.htm }}$ 
En grupos y equipos de trabajo cooperativo, se estudió la composición química de las cajas y las bolsas de leche y se comparó con la información tomada de Internet. Se presentó la siguiente conversación:

Niños: la leche de bolsa y de caja contiene proteínas, vitaminas, hierro, calcio, fósforo, hierro, calorías, azúcares, grasa, sodio, carbohidratos.

Docente: ¿Tiene la misma composición la leche de bolsa y la de caja?

Niños: Sí, y es lo mismo que está en el documento que leímos.

Docente: Entonces ¿qué químico diferente le echan a la leche de caja?

Niños: Ninguno, porque todo es igual en ambas leches. No hay nada diferente, por esto queda descartada la hipótesis uno, ya que la leche de caja como la de bolsa están hechas de lo mismo y su duración no tiene nade que ver con su composición.

Diseño experimental para la hipótesis dos: tomar un empaque de leche en bolsa y uno de caja y comparar los materiales de los que están hechos. Mirar una bolsa y una caja con una lupa para ver si tienen agujeros o si están bien selladas.

Para guiar la actividad el docente preguntó: ¿De qué está hecho el empaque de la bolsa y de caja?

Los niños abrieron cada empaque, los tocaron, compararon y discutieron lo que observaban. Luego, en consenso y con la orientación de los docentes, llegaron a la siguiente conclusión:

"Se encuentran grandes diferencias entre los materiales de los que están hechos los empaques de la leche, por ejemplo, entre el cartón laminado y la bolsa plástica, que influyen en gran medida en el tiempo de duración de la leche y en la calidad de este alimento; es posible que entre aire y luz a través del empaque plástico porque puede venir dañada o rota, por allí se meten los microbios que la dañan; lo contrario ocurre con la caja que tiene más seguridad por su sellado hermético y protección, porque está reforzado con varias capas: aluminio, cartón y plástico para evitar que entren microbios y lo mencionado anteriormente".

Esta hipótesis, conjetura o respuesta no se descarta por parte de los niños.

Además de estudiar la composición de la leche, los estudiantes identificaron en las etiquetas donde se empaca los términos que no conocían, las cuales leyeron y transcribieron en sus libretas de apuntes. Entre estos se tienen: ultrapasteurizada, higienizada, pasteurizada, esterilizada.

Diseño experimental para la hipótesis tres: ir a una empresa procesadora de leche o ver por televisión un programa donde se observe el proceso de pasteurización y los demás, darse cuenta si se hacen procesos a ambos empaques. Se tomó una bolsa y una caja para observarlas en el microscopio para ver si tienen bacterias. Se ponen en refrigeración para mirar cual dura más. 
Se propuso una revisión teórica con una lectura tomada de Wikipedia que tenía relación con los conceptos de pasteurización, ultrapasteurización y esterilización, se elaboró un cuadro comparativo de los procesos de conservación y se trató de responder las siguientes preguntas: ¿Qué es? ¿Por qué lo hacen? ¿Cómo lo hacen? ¿Para qué lo hacen? ¿Cómo lo realizarías en tu casa? ¿A qué conclusión llegamos?

El docente guía de las actividades preguntó si el ultrapasteurizado, higienizados, pasteurizados, esterilizados tendrán que ver con la duración de la leche.

Y los niños respondieron que sí, porque lo hacen las empresas para descontaminar la leche y que cuando ordeñan las vacas lo hacen con las manos sin guantes y pueden estar sucias con microbios; por eso se daña la leche y las empresas la recogen para arreglarla. Con los tratamientos eliminan los microbios.

El docente los invita a revisar la lectura y mirar si lo que dicen es cierto. Los niños en grupo leen el documento y le dieron respuesta a las preguntas que fueron socializadas para poner a prueba las habilidades comunicativas y grupales. El resumen de respuestas se observa en el Cuadro 3.

\section{Cuadro 3. Resultados obtenidos después de la revisión teórica de los procesos de} conservación de la leche.

\begin{tabular}{|c|c|c|c|c|c|}
\hline Preguntas & HTST & UHT & Esterilización & Semejanza & Diferencias \\
\hline ¿Qué es? & $\begin{array}{l}\text { Proceso de } \\
\text { calentamiento } \\
\text { de líquidos, } \\
\text { con el fin de } \\
\text { reducir los } \\
\text { microbios (Mo.) } \\
\text { en los } \\
\text { alimentos. }\end{array}$ & $\begin{array}{l}\text { Proceso de } \\
\text { calentamien } \\
\text { to a ultra } \\
\text { temperatura } \\
\text { s, en el cual } \\
\text { hay } \\
\text { calentamien } \\
\text { to para } \\
\text { eliminar Mo. }\end{array}$ & $\begin{array}{l}\text { Proceso de } \\
\text { eliminación de } \\
\text { toda forma de } \\
\text { vida }\end{array}$ & $\begin{array}{l}\text { Todos } \\
\text { tienen } \\
\text { proceso de } \\
\text { calentamien } \\
\text { to. } \\
\text { Realizado } \\
\text { para evitar } \\
\text { la } \\
\text { contaminaci } \\
\text { ón de los } \\
\text { alimentos }\end{array}$ & $\begin{array}{l}\text { Que no todos } \\
\text { eliminan los Mo., } \\
\text { unos reducen. }\end{array}$ \\
\hline $\begin{array}{l}\text { ¿Cómo lo } \\
\text { hacen? }\end{array}$ & $\begin{array}{l}\text { Se realiza un } \\
\text { proceso } \\
\text { térmico entre } \\
63^{\circ} \mathrm{C} \text { y } 68^{\circ} \mathrm{C} \\
\text { durante } 30 \text { min. } \\
\text { Luego se enfría } \\
\text { a } 4^{\circ} \mathrm{C} \text {. }\end{array}$ & $\begin{array}{l}\text { Se realiza } \\
\text { un proceso } \\
\text { térmico } \\
\text { entre } 135^{\circ} \mathrm{C} \\
\text { y } 150^{\circ} \mathrm{C} \text { en } \\
\text { un tiempo } \\
\text { entre } 2 \text { y } 6 \\
\text { seg. Y luego } \\
\text { se enfría. }\end{array}$ & $\begin{array}{l}\text { Se realiza un } \\
\text { proceso } \\
\text { térmico entre } \\
110^{\circ} \mathrm{C} \text { y } 140^{\circ} \mathrm{C} \\
\text { durante pocos } \\
\text { minutos. }\end{array}$ & $\begin{array}{l}\text { Todos } \\
\text { tienen } \\
\text { proceso de } \\
\text { calentamien } \\
\text { to. } \\
\text { En estos } \\
\text { procesos se } \\
\text { debe } \\
\text { controlar la } \\
\text { temperatura } \\
\text { en relación } \\
\text { al tiempo. }\end{array}$ & $\begin{array}{l}\text { La } \\
\text { pasteurización y } \\
\text { la } \\
\text { ultrapasteurizaci } \\
\text { ón, además de } \\
\text { calentar, se } \\
\text { enfrían, la } \\
\text { esterilización no. } \\
\text { Las } \\
\text { temperaturas en } \\
\text { que se realiza } \\
\text { cada proceso, } \\
\text { son diferentes }\end{array}$ \\
\hline
\end{tabular}




\begin{tabular}{|c|c|c|c|c|c|}
\hline $\begin{array}{c}\text { ¿Para qué lo } \\
\text { hacen? }\end{array}$ & $\begin{array}{l}\text { Para reducir } \\
\text { los Mo. tales } \\
\text { como } \\
\text { bacterias, } \\
\text { hongos } \\
\text { protozoo } \\
\text { levaduras. y }\end{array}$ & $\begin{array}{l}\text { Para } \\
\text { eliminar la } \\
\text { mayor parte } \\
\text { de Mo. a } \\
\text { excepción } \\
\text { de algunas } \\
\text { esporas. }\end{array}$ & $\begin{array}{l}\text { Elimina por } \\
\text { completo } \\
\text { todos los } \\
\text { incluidas las } \\
\text { esporas }\end{array}$ & $\begin{array}{l}\text { Evitan que } \\
\text { los } \\
\text { alimentos } \\
\text { se } \\
\text { descompon } \\
\text { gan y } \\
\text { alargan el } \\
\text { tiempo de } \\
\text { duración de } \\
\text { ellos. }\end{array}$ & $\begin{array}{l}\text { El tipo de } \text { Mo. } \\
\text { que elimina, el } \\
\text { tiempo } \\
\text { duración de } \\
\text { producto. }\end{array}$ \\
\hline $\begin{array}{l}\text { ¿Cómo lo } \\
\text { harías en tu } \\
\text { casa? }\end{array}$ & $\begin{array}{lr}\text { Se pone a } \\
\text { hervir la leche } \\
\text { en una estufa y } \\
\text { con r un } \\
\text { termómetro se } \\
\text { observa la } \\
\text { temperatura en } \\
\text { que r se } \\
\text { encuentra la } \\
\text { leche, cuando } \\
\text { esté en la } \\
\text { temperatura y } \\
\text { tiempo } \\
\text { indicado, se } \\
\text { apaga y se } \\
\text { mete } \\
\text { rápidamente a } \\
\text { un congelador. }\end{array}$ & $\begin{array}{l}\text { Se busca } \\
\text { una estufa o } \\
\text { un horno } \\
\text { microondas } \\
\text { que pueda } \\
\text { medir } \\
\text { grados de } \\
\text { temperatura } \\
\text { se coloca } \\
\text { la leche y se } \\
\text { deja el } \\
\text { tiempo y la } \\
\text { temperatura } \\
\text { indicada, } \\
\text { luego se } \\
\text { guarda en } \\
\text { un congelador. }\end{array}$ & $\begin{array}{l}\text { Se busca una } \\
\text { estufa que } \\
\text { mida los } \\
\text { grados de } \\
\text { temperatura, } \\
\text { se calienta la } \\
\text { leche en el } \\
\text { tiempo y } \\
\text { temperatura } \\
\text { sugerida, se } \\
\text { deja reposar y } \\
\text { se guarda en } \\
\text { la nevera. }\end{array}$ & $\begin{array}{l}\text { Que todos } \\
\text { tienen el } \\
\text { proceso de } \\
\text { calentamien } \\
\text { to y se hace } \\
\text { a nivel, } \\
\text { artesanal } \\
\text { (casa) }\end{array}$ & $\begin{array}{l}\text { Que todos los } \\
\text { procesos tienen } \\
\text { diferente } \\
\text { temperatura y } \\
\text { tiempo para } \\
\text { realizarse. }\end{array}$ \\
\hline $\begin{array}{l}\text { A que } \\
\text { conclusión se } \\
\text { llegó. }\end{array}$ & \multicolumn{5}{|c|}{$\begin{array}{l}\text { Que si a la leche se le hacen procesos de pasteurización, ultrapasteurización o } \\
\text { esterilización, se conserva por más tiempo, alargando la duración de la misma } \\
\text { debido a que se evita los microbios en la bolsa y en la caja. }\end{array}$} \\
\hline
\end{tabular}

Al finalizar la actividad surgieron algunas preguntas, como si a ambas leches se le hacían todos los procesos, y se concluyó que existen diversas técnicas de conservación de la leche durante períodos prolongados, que se hacen, tanto a la leche de caja como la de bolsa, como son: pasteurización, ultrapasteurización, esterilización. Entonces, no es por esto que dura más la leche de caja que la de bolsa, se descarta la hipótesis.

Plantear una nueva situación problema como mecanismo de valoración: para establecer, si hubo aprendizaje significativo, se planteó una situación donde se aplicaran los conceptos estudiados en una situación real.

En la granja Santa Lucía, propiedad de la Universidad de la Paz, se producen diariamente 2.000 litros de leche, que es recogida en carros cisternas y llevada hasta las empresas cercanas para su procesamiento. Un día el carro no pasó a recoger la leche y los campesinos se preocuparon porque la leche se dañaría, generándoles grandes pérdidas económicas. De acuerdo con la situación, plantea la(s) pregunta(s) problema(s) que 
queremos resolver; formula una o varias hipótesis, conjeturas o respuestas a la pregunta formulada; plantea un diseño o soluciones a la situación, predice los resultados y anota tus conclusiones.

Las preguntas problema planteadas por los niños fueron:

1. ¿Qué deben hacer los campesinos para que la leche no se les dañe?

2. ¿Cómo deben conservar la leche los campesinos para que no se les dañe?

3. ¿Qué método de conservación deben aplicar los campesinos a la leche para que no se les dañe y dure por más tiempo?

De igual manera, plantearon las siguientes hipótesis:

1. Si hierven la leche, la tapan y la ponen en un lugar fresco no se les daña.

2. Si hierven la leche, la tapan, la dejan reposar, la meten a la nevera no se les daña.

3. Si se hace un método de pasteurización casero la leche se conserva por más tiempo.

Y plantearon los siguientes diseños:

\section{Primer diseño}

1. Tomar la olla donde se hacen los sancochos (comida típica de la región) y hervir los 2.000 litros de leche.

2. Dejar la leche reposar un rato en un recipiente tapado para que se enfríe.

3. Guardarla en la nevera.

\section{Segundo diseño}

1. Tomar una olla grande y hervir los 2.000 litros de leche en el fogón de leña.

2. Dejar la leche reposar un rato y sacarle la nata.

3. Tapar bien la leche y ponerla en un lugar que no sea muy caluroso.

\section{Tercer diseño}

1. Tomar una olla grande y hervir los 2.000 litros de leche a una temperatura de $63-65^{\circ} \mathrm{C}$, midiéndola con un termómetro, durante 30 minutos.

2. Dejar la leche reposar un rato en un recipiente tapado y meterla a uno más grande con hielo para bajarle rápido la temperatura.

3. Ponerla tapada en un lugar fresco.

Como predicción indicaron que la leche se conserva por más tiempo. Si se hace bien el proceso no se dañará tan rápido porque no le entrarán microbios. Los campesinos podrán vender la leche y no tendrán pérdidas económicas.

Y concluyeron que se pueden hacer métodos de pasteurización caseros, calentándola en la estufa y enfriándola en la nevera. Así se matarían los microbios y para evitar que otros entren a la leche, se debe guardar la leche en recipientes herméticamente cerrados y libres de microbios. 


\section{Resultados y discusión}

- Al manejar reiteradamente los conceptos científicos, en este caso el informe de la composición de la leche y comparados con lo impreso en las etiquetas de la caja y la bolsa, los niños se dieron cuenta de que a la leche en caja no se le agrega ningún químico diferente a los que se echan a la de bolsa. Ambas leches presenta los mismos componentes. Por lo tanto la hipótesis uno se descarta y surge el interrogante ¿qué será entonces lo que hace que la leche de caja dure por mucho tiempo?

La actividad le permitió a cada niño autorreflexionar y explicitar a partir de la cuestión qué habían hecho hasta el momento. En consenso se llegó a la siguiente explicación: "En clases anteriores se formularon tres hipótesis para responder a la pregunta ¿por qué la leche en caja dura más que la de bolsa? Posteriormente plantearon diseños experimentales para demostrar cuál era la más acertada; concluyeron que la leche en caja dura más que la de bolsa por el empaque, por esto la hipótesis correcta fue la número dos. La discusión sobre la actividad realizada, les dio herramientas y estrategias a los estudiantes para organizar su pensamiento. Al reflexionar sobre su aprendizaje, pudieron visualizar sus objetivos y las estrategias de acción que debían desarrollar para alcanzarlos, obteniendo así conocimiento científico escolarmente construido.

Por lo tanto la autorregulación de aprendizaje constituye un aporte a la adquisición de habilidades cognitivas superiores (uso del pensamiento crítico y lógico) y a la propia construcción de conocimientos, en la medida en que les sirve para planear, controlar y evaluar el desarrollo que tienen sobre las responsabilidades y tareas que deben realizar.

- Los problemas se trataron siguiendo una orientación científica, con emisión de hipótesis y explicitación de las ideas previas, elaboración de estrategias posibles de resolución que fueron revisados para seleccionar los más viables, teniendo en cuenta que se ajustaran a las hipótesis formuladas y pudieran desarrollarse en el aula de clase, de tal modo que permitieran poner a prueba los conocimientos que se construyeron hasta el momento. La efectividad en la resolución de un problema no solo depende de los conocimientos básicos, sino también de un procedimiento adecuado que facilite la búsqueda de una solución.

- La construcción de conocimiento científico escolar estuvo mediada por la interacción e influencia de los demás (practicantes/compañeros), a través de la estrategia de aprendizaje cooperativo que se puso en práctica en pequeños grupos de trabajo. Cada estudiante desempeñaba una función bien definida en las actividades programadas: hablar, preguntar, escribir, escuchar, lo que permitió una participación activa de los niños y niñas.

En estos grupos se negociaron, discutieron e intercambiaron ideas, significados, conocimientos cotidianos, que evolucionaron porque se propició la explicación de los propios puntos de vista, la constatación con otros y se hicieron compatibles con aspectos conceptuales de mayor complejidad. La responsabilidad por el progreso de cada equipo de trabajo fue asumida por todos sus miembros, logrando de este modo metas compartidas que 
tuvieron un efecto favorable en el rendimiento académico y las relaciones socio-afectivas del curso, ya que el aprendizaje constituye una construcción eminentemente social.

En la interacción entre grupos de trabajo cooperativo, conformados por un número de 4 a 6 niños, todos colaboraron en el aprendizaje. Cada niño asumió su responsabilidad para desarrollar una acción. Para ello se pusieron de acuerdo sobre lo que haría cada uno, distribuyéndose roles como leer, escuchar, tomar apuntes de las ideas principales de la lectura y discutir sus puntos de vista. De esta manera alcanzaron los objetivos propuestos, con la ayuda de los practicantes que dirigieron y supervisaron el proceso.

El aprendizaje en equipos de trabajo cooperativo implica un concepto de aprendizaje no competitivo ni individualista, sino un mecanismo colaborador que permite a los niños desarrollar hábitos de trabajo en equipo para participar activamente en la construcción colectiva, asumir y cumplir compromisos grupales, contrastar sus actividades y creencias con las de los demás, exponer sus ideas y planteamientos en forma argumentada y poner en práctica valores como la solidaridad entre compañeros, tolerancia, respeto y desarrollar habilidades interpersonales.

La evaluación, por su parte, se constituyó en un instrumento de formación donde los estudiantes participaron en la regulación de su aprendizaje y detectaron sus niveles de formación y destrezas, debilidades, fortalezas, la forma en que podían revisar y rectificar concepciones erróneas y asumir decisiones reflexivas y críticas.

Con las actividades se pretendía formar personas conscientes de su capacidad de aprendizaje, que pudieran trabajar los problemas que la realidad les plantea, actuar reflexiva e inteligentemente ante diversas situaciones vitales y fueran capaces de regular sus propios procesos de aprendizaje y ponerlos al servicio de la sociedad.

En una situación de aprendizaje orientada a la construcción de conocimientos y a la investigación, los niños compararon sus producciones con las de otros equipos, valorando sus trabajos y buscando la orientación de los docentes para recibir la ayuda necesaria, a través de la lectura de informes que les permitieron darse cuenta que a la leche de caja no le hacen procedimientos distintos a los de la leche de bolsa; tienen los mismos procesos de conservación y que no es esto lo que hace que dure por más tiempo.

En esta actividad fue fundamental llevar al aula de clase material concreto que permitiera a los estudiantes analizar, establecer comparaciones y argumentar si la hipótesis 2 se descartaba o no; aquí el aprendizaje dejó de ser una cuestión individual y se convirtió en una tarea de equipo en la que los niños participaron en la resolución de la actividad. Esto sirvió para que se dieran cuenta de que la caja está hecha de un material más resistente que el de la bolsa y que además está sellada herméticamente, por esto la leche se conserva por más tiempo.

Se insistió en la necesidad de que los niños desempeñen un papel más activo en clase, en tareas diversas, desde realizar experiencias hasta resolver problemas, lo que constituye una alternativa a la memorización simple de los conocimientos.

- Los nuevos modelos científicos escolares que se van configurando a partir de las preguntas y explicaciones, puestas en el aula de clase, sirvieron para que los niños reconocieran la utilidad de los conocimientos adquiridos para transferirlos a su diario 
vivir. También para darse cuenta de su nivel de capacitación y formación que les permite continuar estudios más avanzados en ciencias, en la búsqueda de acceder a herramientas necesarias en la solución de problemas de su entorno. De igual manera para comprobar que el ejercicio también funcionan en la toma de decisiones.

- El aprendizaje basado en problemas, enfrenta a los niños a situaciones a las que se debe dar solución. Esta estrategia hace explícita la aplicación de los conocimientos aprendidos a situaciones problemáticas nuevas, fomentando la utilidad de los mismos, existiendo una interrelación continua entre teoría y aplicación práctica, integrando los conocimientos conceptuales, procedimentales, actitudinales y axiológicos.

La experiencia en clase enseña que entre las numerosas habilidades que se requieren para construir y desarrollar la cultura del conocimiento científico escolar, se hace necesaria la creación de una comunidad de indagación donde el aprendizaje y la enseñanza de las ciencias se den como un proceso de (re)construcción de conocimientos. En un contexto que se inspire en la metodología de la investigación como forma de favorecer, tanto una actividad significativa en torno a problemas susceptibles de interesar a los estudiantes, como en su progresiva autonomía de juicio y capacidad de participación en tareas colectivas. De este modo escucharíamos de los niños y los docentes, la clase de respuestas, preguntas, hipótesis, reflexiones y explicaciones que reflejarían la naturaleza de la cultura del conocimiento científico escolar. En este espacio no se teme modificar puntos de vistas o corregir algún razonamiento - los propios o los de compañeros, que parezcan defectuososSe está dispuesto a abandonar una idea o una respuesta que se considere deficiente.

El éxito del aprendizaje parece estar originado a través de la reflexión sobre el significado, la identificación y resolución de contradicciones. Conexiones entre pensamientos y palabras, por un lado, y acciones por el otro. Incorporar la resolución de problemas es pensar tanto en forma crítica como creativa, haciendo preguntas esclarecedoras y tomando en consideración posibilidades de soluciones alternativas. Reflexionar sobre las creencias personales (idea actual) y la información que se propone (nuevas ideas), es parte de la clave de la consciencia y control sobre el propio aprendizaje.

\section{Conclusiones}

- Los docentes en edades iniciales, al formular las preguntas, deben tener en cuenta tres aspectos fundamentales para su eficacia:

La forma, que se refiere al modo de expresar la pregunta, es decir el carácter abierto o cerrado de la misma y su enfoque relativo a la disciplina o a la persona. Las preguntas abiertas dejan más opciones para responder (¿Por qué? ¿Qué pasaría si?), que las cerradas (¿Qué? ¿Cuándo? ¿Dónde? ). Cuando las preguntas centradas en las personas, se expresan de manera que inviten al niño a exponer sus ideas sin tener en cuenta que unas sean más acertadas que las otras (¿Qué crees?), en cambio de las preguntas centradas en la disciplina, que se refieren a los contenidos de un modo que indica la existencia de una respuesta correcta (¿Qué hay dentro de?).

El momento, es el segundo aspecto que hay que tener en cuenta en el planteamiento de la pregunta. Esto significa que en el principio de un tema, se pregunta para indagar las ideas que tienen los niños sobre el contenido a desarrollar. Como actividad de exploración, se puede utilizar preguntas de este tipo: ¿Dónde crees? ¿Por qué crees? ¿Qué crees que? 
¿Puedes decir por qué? ¿Cómo puedes comprobar? ¿Qué pasaría si...? ¿Puedes encontrar un modo de...? ¿Cómo harías para que...?

Y el tercer aspecto es la pregunta que desarrolle determinadas destrezas en una investigación que estimule la observación, elaboración de hipótesis, la predicción, el diseño, las conclusiones y la comunicación: ¿En qué se parecen? ¿Qué diferencias? ¿Qué ocurre cuando? ¿Por qué crees? ¿Qué crees que? ¿En qué crees que? ¿Qué podríamos hacer? ¿Qué tendrías que hacer? ¿Cómo puedes asegurarte? ¿Qué herramientas necesitas? ¿Encuentras alguna relación? ¿Qué cosas influyen? ¿Cómo vas a tomar? ¿Cómo lo puedes explicar? ¿De qué manera lo representas?

- Un niño curioso quiere conocer, probar experiencias nuevas, explorar, descubrir aspectos relativos a su entorno. La curiosidad se muestra a menudo en forma de preguntas, pero este no es el único signo de curiosidad ni el único síntoma que estimular. Efectivamente, invitar a que los niños pregunten es una de las formas de mostrar que valoramos la curiosidad y la actitud puede favorecerse de este modo, si el resultado es satisfactorio e interesante para ellos.

- Hacer preguntas proporciona una satisfacción que ayuda a los niños a compartir sus alegrías y su entusiasmo con los demás, porque forma parte del curso natural del desarrollo, y menospreciarlas puede contribuir a que la curiosidad se atenúe. Por otra parte, la satisfacción que logran al expresar su curiosidad, ayuda a que accedan a una etapa más madura en la que el interés se mantenga durante más tiempo y las preguntas sean más reflexivas.

- $\quad$ Es importante que las actividades implementadas por los docentes en estas edades, den a los niños, a partir de las preguntas, la oportunidad para desarrollar las ideas básicas sobre el mundo circundante. Por ello es necesario que sean interesantes y despierten su curiosidad, que se relacionen con sus experiencias cotidianas y les ayuden a comprenderla. Que le den la oportunidad para implementar técnicas de procedimientos en la resolución de problemas, promover las actitudes científicas como la curiosidad para hacer preguntas, querer saber más. Que sean flexibles para replantear las ideas y reconocer que estas son provisionales. Que promuevan la reflexión crítica para reconocer y reconsiderar los métodos utilizados, el deseo de perfeccionar las ideas y sensibilidad con respecto a los seres vivos y al medio ambiente.

Según Perkins (1986), un conocimiento activo es el que puede aplicarse y requiere cuatro elementos: una pregunta, una estructura de conocimiento en la cual tenga sentido la pregunta, ejemplos de cómo responder la pregunta y una argumentación que estructure la respuesta.

Esta definición es coherente con otras aportaciones (Guidoni, 1985) y con lo que se ha venido diciendo respecto a la actividad científica: conocimiento, lenguaje, cultura (valores y aplicaciones) deben relacionarse, y solo así dan lugar a acciones humanas. Todo ello proporciona una clara orientación con relación a lo que debería ser la clase:

- $\quad$ Generadora de preguntas, y por ello vinculada a las ideas de los alumnos y a su propia visión del mundo.

- $\quad$ Estructuradora de conocimiento, y por ello encargada de enseñar a pensar sobre el mundo mediante modelos, para generar hechos científicos y teorías. 
- Transformadora del mundo, y por ello conectada con las aplicaciones del conocimiento estructurado, que solo así adquirirá sentido.

- Argumentadora, porque el conocimiento científico es, finalmente, conocimiento escrito, y gracias al lenguaje disponemos de representaciones del mundo que lo hacen "explicable" e inteligible.

La evaluación se constituyó en un instrumento de formación. Los niños participaron en la regulación de su aprendizaje a través de ejercicios de autorregulación que fueron clave para la toma de conciencia y control de sí mismos. Reflexionaron acerca de sus conocimientos cotidianos iniciales y la forma en que habían evolucionado o complejizado sus ideas, reconociendo y valorando sus fortalezas, dificultades y rectificando sus errores

\section{BIBLIOGRAFÍA}

ANIBAL, N., J. (1989). Preguntario. Tres Cultura Editores. Universidad Inca- Colombia. p. 27.

BACHELARD, G., (1982) La formación del espíritu científico, Siglo XXI, México

COLL, C., La conducta experimental en el niño, CEAC, Barcelona, 1978.

-, "Estructura grupal, interacción entre alumnos y aprendizaje escolar", en Infancia y Aprendizaje, núms. 27-28, 1984, pp. 119-138.

DAZA, S. Y ARRIETA, J. (2006): Los conceptos sobre ciencia y trabajo científico y sus implicaciones en la elaboración de los programas de ciencias naturales. Revista TED, 20. P. 80-95. Bogotá: Universidad Pedagógica Nacional.

DRIVER, R., SQUIRES,A., RUSHWORTH, P. Y WOOD-ROBINSON, V.(1999). Dando sentido a la ciencia en secundaria. Investigaciones sobre la ideas de los niños. MadridEspaña. Visor. P. 24.

DUSCHL, R., (1995), Más allá del conocimiento: los desafíos epistemológicos y sociales de la enseñanza mediante cambio conceptual, enseñanza de la ciencia 13 (1), 3-14.

DUCKWORTH, E. (1999). Cuando surgen ideas maravillosas. Y otros ensayos sobre enseñanza y el aprendizaje. Barcelona - España. Gedisa editores. p. 23-26.

ELDER, L Y PAUL, R. (2002) El arte de formular preguntas esenciales. Foundation for Critical Thinking. P2

http://www.eumed.net/libros/2010b/708/LAS\%20GRANDES\%20PREGUNTAS.htm

GIL, D; VILCHES, A; TOSCANO, C Y MACIA. O. (2006). Década de la educación para un futuro sostenible (2005-2014): un punto de inflexión necesario en la atención a la situación del planeta. Revista Iberoamérica de Educación. № 40.125-178.

GIL, D; CARRASCOSA, J. y MARTÍNEZ, TORREGROSA J. (1991) La enseñanza de las ciencias en la educación secundaria. Barcelona: Horsori. 232. 
GIL, D. y PESSOA, de Carvalho A. (2000). Dificultades para la incorporación a la enseñanza de los hallazgos de la investigación e innovación en didáctica de las ciencias. Educación en Química, 11 (2). $244-251$.

GUIDONI P. (1985). “On natural thinking”, European Journal of Science Education, 7, 133140.

HARLEN, W. (1999). Enseñanza y aprendizaje de las ciencias. Madrid- España. Ediciones Morata. p. 97-112.

MARTINEZ-TORREGROSA, J., GIL D., Y MARTINEZ, B. (2003) La universidad como nivel privilegiado para un aprendizaje como investigación orientada. En: MONEREO C, OSBORNE, R Y WITTROCK, M. (1983).Learning science: a generative process. Science Education, 67, 490-508.

OGBORN, J., KRESS, G. y MCGILLICUDDY, K. (1998). Formas de explicar. La enseñanza de las ciencias en secundaria. Madrid-España. Aula XXI, Santillana. p. 45.

PERKINS D.N. (1986). Knowledge as design, Hillsdale, Erlbaum Associates.

PERRET-CLERMONT, A.N., "Perspectivas psicosociológicas del aprendizaje en situación colectiva", en Infancia y aprendizaje, núm. 16, 1981, pp. 29-41.

POZO, J,I (2003) (Comps.).La universidad ante la nueva cultura educativa. Enseñar y aprender para la autonomía. Ed. Síntesis.231-244.

QUINTANILLA, M. (2006) Identificación, caracterización y evaluación de competencias científicas desde una imagen naturalizada de la ciencia. En: Quintanilla y Adúriz-Bravo, (Ed.), Enseñar ciencias en el nuevo milenio. Retos y desafíos. (pp.18-42) Santiago, Chile: Pontificia Universidad Católica de Chile.

SAEZ, J y RIQUARTS K. (2002). Educación científica para el desarrollo sostenible, En Membiela, P (Comps.), enseñanza de las ciencias desde la perspectiva ciencia-tecnologíasociedad formación científica para la ciudadanía. Ed. Narcea. 47-59. 